\title{
Validation of SPECT-CT image reconstruction for the Mediso AnyScan SCP scanner in STIR
}

\author{
Daniel Deidda, Member, IEEE, Benjamin A. Thomas, Kelley Ferreira, Warda Heetun, Attila Forgács, Brian F. \\ Hutton, Senior Member, IEEE, Kris Thielemans, Senior Member, IEEE, Andrew P. Robinson
}

\begin{abstract}
Single photon emission tomography (SPECT) is widely used in clinical practice for a large number of diagnostic applications. However, different hospitals might use different scanners containing different hardware and software technologies, therefore making reproducibility of results a hard task. The aim of this work is to establish the basis for a tool able to include different clinical scanner models to facilitate inter-comparison of activity measurements across different institutions. We used the open source software for tomographic image reconstruction (STIR) and implemented functionalities to read, process and reconstruct the data from the Mediso AnyScan SCP scanner. In particular, the triple energy window method for scatter estimation, a method to re-scale the CT Hounsfield units in attenuation coefficient units $\left(\mathrm{cm}^{-1}\right)$, SPECT normalisation functionalities and list mode (LM) functionalities were implemented and tested. 3D printed phantom data, with organ inserts filled with ${ }^{177} \mathrm{Lu}$, and a ${ }^{99 m}$ Te clinical bone study were reconstructed using the implemented corrections and different anatomically-guided algorithms. The effect of the aforementioned correction was studied using ROI analysis and line profiles, whereas visual comparison was carried out between the reconstructed images with the vendor software and with STIR. Moreover, we demonstrated the feasibility of SPECT image reconstruction using the $\mathrm{CT}$ and iterative SPECT image estimates as prior information. Finally, a run time performance study showed that, when using multiple cores, an acceleration of a factor 2.7 is achieved for OSEM and around 2 for the other algorithms which involved more image-based operations. The availability of such tool will make SPECT research applications more accessible and reproducible.
\end{abstract}

\section{INTRODUCTION}

$\mathbf{S}$ PECT combined with CT is nowadays routinely used in clinical practice in a wide range of applications [1,2]. A number of commercial scanners are available on the market and different models are used in different hospitals. In addition, different regularisation, system models and reconstruction methods might be implemented in each model, making studying reproducibility of quantitative measures challenging.

A freely available independent tool able to include different scanner models is necessary to compare measurements across

Manuscript received December, 10, 2019. This work is supported by the UK National Physical Laboratory through the National Measurement System, and the joint research project (JRP), metrology for clinical implementation of dosimetry in molecular radiotherapy (MRTDosimetry), within the European Metrology Programme for Innovation and Research (EMPIR) (http://mrtdosimetry-empir.eu/)

D. Deidda, (e-mail: daniel.deidda@npl.co.uk), K. Ferreira, W. Heetun and A. Robinson are with the Division of Nuclear Medicine, Medical Radiation Physics, National Physical Laboratory, Teddington, UK;

B. A. Thomas, B. F. Hutton, K. Thielemans, D. Deidda are with the the Institute of Nuclear Medicine, University College of London, UK;

A. Forgács is with with ScanoMed Ltd, Debrecen, Hungary. different institutions and allow traceability of measured activity. To establish the basis for this activity measure intercomparison, we extended the 3D SPECT modelling [3] in the open source software for tomographic image reconstruction (STIR) [4] to model the Mediso AnyScan SPECT-CT-PET (SCP). We therefore present a performance study of the SPECT functionality implemented in STIR for the Mediso AnyScan SCP and we compare reconstructed images using a series of reconstruction methods.

\section{Methods AND Material}

\section{A. Implemented Utilities}

Two different way of applying the TEW method were used: the first subtracts the TEW sinogram from the peak sinogram prior to the reconstruction and the result is thresholded to avoid negative values. The second includes the TEW sinogram as an additive term in the forward model.

Attenuation correction (AC) is performed from the lowdose CT image by rescaling the CT values to the appropriate attenuation coefficient values for a given energy [5].

An effort was made to implement LM functionalities for SPECT. Traditionally, the base class, CListModeData, to read and process LM data in STIR was designed for coincidence events, therefore for PET. In this work, we extended STIR such that we have a more generic base class, ListModeData where CListModeData is derived from. SPECTListModeData, was then created as a derived class for SPECT LM data (Figure II-A). In this way, the PET scanner models do not need to be modified but we can add SPECT scanner models as derived classes from SPECTListModeData. The scanner model for the Mediso AnyScan SCP was implemented and tested.

Since we want to be able to reproduce every step from the source preparation to the image reconstruction, a way of applying corrections such as uniformity and decay was developed. This follows the same idea of the normalisation bin used for PET. These two corrections can be applied during the reconstruction or after the binning from list mode (LM) to sinogram. The decay correction is calculated with the exponential decay law and changes according to radioisotope and duration of acquisition.

The uniformity table is estimated during the calibration protocol. The table is read by STIR and each factor applied to the sinogram.

Another calibration table that we are able to apply is the center of rotation $(\mathrm{CoR})$ which allows the center of the image to coincide with the centre of the scanner. Currently this correction is applied event by event from the LM class. 

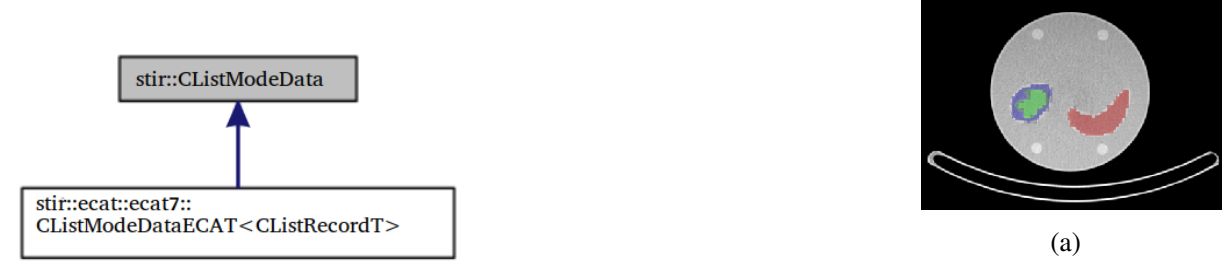

(a)

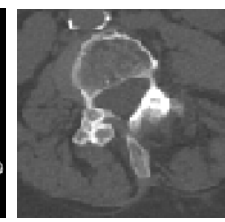

(b)

Fig. 2. CT image with the chosen ROIs for the phantom (a), and for the patient (b)

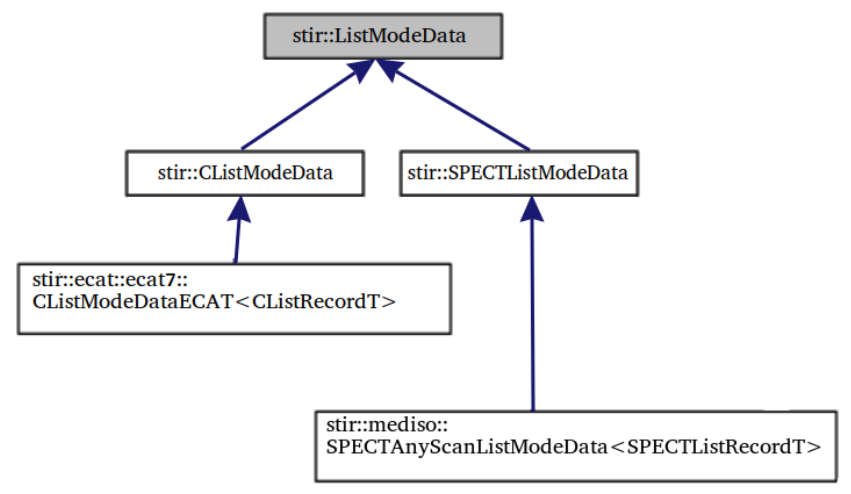

Fig. 1. The new list mode hierarchy

\section{B. Experiments}

The data has been acquired using the Mediso AnyScan SCP. The PET camera was not used for this study. The SPECT camera consists of three heads that can be set at different angular configurations. The spatial resolution with scatter using a low energy high resolution (LEHR) at $10 \mathrm{~cm}$ is $7.3 \mathrm{~mm}$. The energy range is $40-960 \mathrm{keV}$. Each head consists of a $\mathrm{NaI}(\mathrm{Tl})$ crystal with $9.5 \mathrm{~mm}$ thickness. Behind the detector there is an array of 94 photomultiplier (PMT). The detector useful field of view (FOV) is $560 \times 425 \mathrm{~mm}$. The patient aperture is 75 $\mathrm{cm}$. The CT gantry has $50 \mathrm{~cm}$ FOV with a patient aperture of $70 \mathrm{~cm}$. The X-ray detector is a ceramic detector consisting of $24 \times 896$ elements. The X-ray tube voltage spans from $80 \mathrm{kV}$ to $140 \mathrm{kV}$ with a current up to $500 \mathrm{~mA}$.

The acquired data were pre-corrected for detector linearity, uniformity and center of rotation (COR) using the vendor software. Scatter estimates for scatter correction (SC) were obtained selecting events via the triple energy window (TEW) method [6,7], using $20 \%$ width for the peak window and $6 \%$ for the upper and lower windows.

\section{Phantom and patient Data}

The phantom used in this work has been acquired at the National Physical Laboratory (NPL) and was designed within the molecular radiotherapy dosimetry project (http://mrtdosimetryempir.eu/). It contains 3D printed inserts representing a spleen and a kidney allowing cortex and medulla to be injected with different activities. The injected ${ }^{177} \mathrm{Lu}$ activities are: $185.1 \pm 0.9$ $\mathrm{MBq}$ for spleen, $111.6 \pm 0.6 \mathrm{MBq}$ for the kidney cortex, and $22.8 \pm 0.1 \mathrm{MBq}$ for the kidney medulla. The ${ }^{177} \mathrm{Lu}$ energy spectrum is characterised by two gamma peaks, 113 and 208 $\mathrm{keV}$. In this study, only the $208 \mathrm{keV}$ peak is selected, as it is the one used for dosimetry purposes. The data were acquired over 60 minutes and total number of detected events is $8.95 \times 10^{6}$.

The patient data, from a ${ }^{99 m} \mathrm{Tc}$ bone scintigraphy study, were acquired and anonymised with the same scanner model at the department of nuclear medicine, ScanoMed OD KFT, Debrecen, Hungary. The injected activity was $509.5 \mathrm{MBq}$ and it was acquired for $30 \mathrm{~min}$.

\section{Reconstruction Setup}

To provide a demonstration of the possible choices available, all the datasets were reconstructed with 20 iterations and 12 subsets, using the OSEM with Gaussian post-filter and the latest implemented anatomically-driven reconstruction algorithms: OSMAPOSL with the parallel level sets (PLS) prior [8], the kernelised expectation maximisation (KEM) [9] and the hybrid KEM (HKEM) [10, 11, 12]. The image size is $128 \times 128 \times 96$ voxel size, while the voxel size is $4.2578 \times 4.2578 \times 4.2578 \mathrm{~mm}^{3}$. Images with the same settings were reconstructed with the vendor software using OSEM for comparison.

\section{Images Analysis}

The analysis was carried out using segmented regions from the CT image, as reported in Figure 2, to calculate the mean value in the spleen, cortex and medulla. The Mean was calculated as follows:

$$
t=\frac{\sum_{j=1}^{V} t_{j}}{V}
$$

where $t$ is the mean value of the target ROI, $t_{j}$ is the value of voxel $j$ within the ROI, and $V$ is the number of voxels within the ROI. Line profiles were also extracted to appreciate the voxel-wise variation between images reconstructed with and without scatter and AC.

\section{RESUlTS AND DISCUSSION}

The effect of two different methods for taking scatter into account was studied. Figure 3(a) shows the effect due to the two different ways of applying SC. As we can see, both provide a similar reduction of activity concentration in the three chosen ROIs. However, as expected modelling the scatter in the forward model provides a slightly higher reduction, this is due to the fact that when the sinogram is pre-corrected a threshold 
has to be applied to remove negative values leading to a loss of information. In addition, from the same figure is possible to notice that for all ROI the mean value has reached a plateaux after 4 iterations.

Figure 3 reports a comparison between line profiles on the image reconstructed using no correction, only SC (modelled as additive term), only $\mathrm{AC}$, and both $\mathrm{SC}$ and $\mathrm{AC}$. It is visible that the most significant effect is obtained when $\mathrm{AC}$ is applied. In fact, it can be seen that the profile is more defined and there is a increase in activity concentration. Figure 4 provides a demonstrative comparison between the images reconstructed with OSEM using the vendor software and STIR, as well as a demonstration that it is possible to use, for SPECT-CT data (both phantom and patient), some of the state-of-the-art anatomically-driven algorithms which were proposed in the context of PET-MR. Figure 5 shows the sinograms obtained from the LM data, and compares between the vendor software and STIR. The difference in the total number of counts was around $2 \%$ which is due to corrections that are not currently implemented in STIR, such as dead time.

Finally, Table I shows the run time acceleration achievable when using 10 cores and the message passing interface (MPI) technology. It can be seen that OSEM can achieve 2.7 acceleration factor, whereas the factor gradually decreases for the other methods until 1.6 for HKEM. This is due to the fact that the parallelisation is performed at the projection level and the CT-driven algorithms have more image-based operation than OSEM.

The availability of this tool will have potential implications in different areas, such as molecular dosimetry and metrology. In addition, when the Mediso AnyScan PET camera will be added in STIR it will be possible to conduct studies with triple modality datasets.

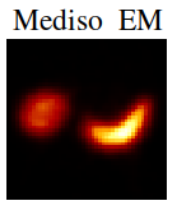

Mediso EM

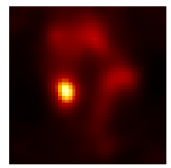

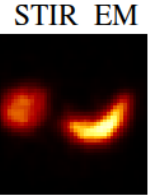

STIR EM

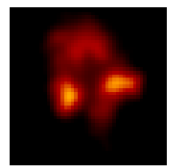

STIR PLS

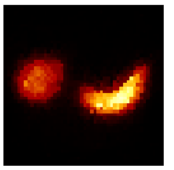

STIR PLS

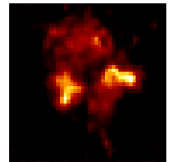

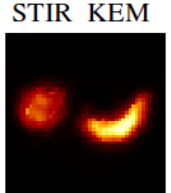

STIR KEM

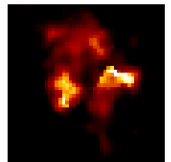

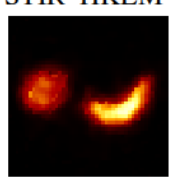

STIR HKEM

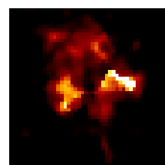

Fig. 4. Reconstructed images with OSEM followed by Gaussian postfilter, OSEM-PLS, KEM and HKEM: top row for OSEM using the Mediso software on the right side, and OSEM using STIR on the left; the CT-driven reconstructed images with the PLS prior, KEM and HKEM in the bottom row. Top row: phantom data; Bottom row: patient data.

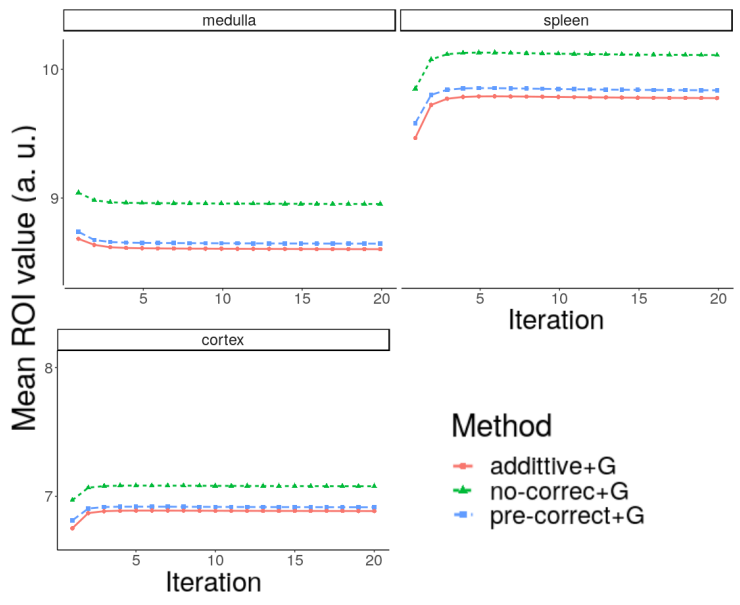

(a)

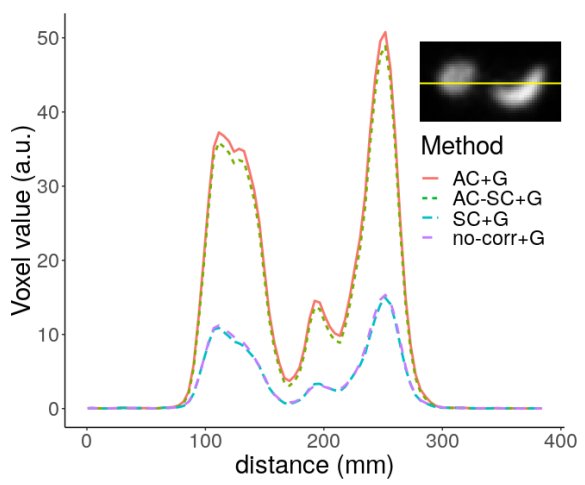

(b)

Fig. 3. Comparison between reconstructed images using OSEM with no correction, only AC, only SC and both AC and SC. (a) Compares no corrected images with pre-corrected SC, and SC modelled as additive term, using 20 full iterations and 3 ROIs; (b) the plot shows the line profile for $\mathrm{AC}, \mathrm{AC}+\mathrm{SC}, \mathrm{SC}$ and no corrections. All the images reconstructed with OSEM have been postprocessed using a Gaussian filter.

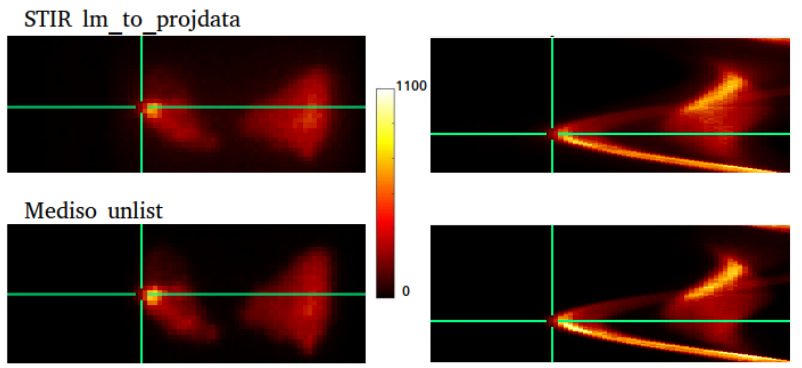

Fig. 5. Comparison between the unlisting utilities from STIR and Mediso 
TABLE I

RUN TIME PERFORMANCE (S) FOR THE PHANTOM RECONSTRUCTED IMAGES WITH 20 FULL ITERATIONS INCLUDING TEW-SC AND AC.

\begin{tabular}{lcc}
\hline & 1 core & 10 cores \\
\hline OSEM & 676 & 246 \\
PLS & 773 & 302 \\
KEM & 1114 & 549 \\
HKEM & 1209 & 768 \\
\hline
\end{tabular}

\section{WORK IN PROGRESS}

We are currently working on the validation of all the created functionalities within STIR. A similar implementation will be provided for other scanners in the future.

\section{REFERENCES}

[1] S. Arvola, I. Jambor, A. Kuisma, J. Kemppainen, S. Kajander, M. Seppänen, and T. Noponen, "Comparison of standardized uptake values between $99 \mathrm{~m} \mathrm{Tc}$-HDP SPECT/CT and $18 \mathrm{~F}-\mathrm{NaF}$ PET/CT in bone metastases of breast and prostate cancer," EJNMMI research, vol. 9, no. 1, p. 6, 2019.

[2] D. L. Bailey and K. P. Willowson, "An evidence-based review of quantitative SPECT imaging and potential clinical applications," Journal of Nuclear Medicine, vol. 54, no. 1, pp. 83-89, 2013.

[3] B. M. Fuster, C. Falcon, C. Tsoumpas, L. Livieratos, P. Aguiar, A. Cot, D. Ros, and K. Thielemans, "Integration of advanced 3D SPECT modeling into the open-source STIR framework," Medical physics, vol. 40, no. 9, p. 092502, 2013.

[4] K. Thielemans, C. Tsoumpas, S. Mustafovic, T. Beisel, P. Aguiar, N. Dikaios, and M. W. Jacobson, "STIR: software for tomographic image reconstruction release 2," Physics in Medicine and Biology, vol. 57, pp. 867-883, 2012.

[5] S. Brown, D. L. Bailey, K. Willowson, and C. Baldock, "Investigation of the relationship between linear attenuation coefficients and CT hounsfield units using radionuclides for SPECT," Applied Radiation and Isotopes, vol. 66, no. 9, pp. 1206-1212, 2008.

[6] T. Ichihara, K. Ogawa, N. Motomura, A. Kubo, and S. Hashimoto, "Compton scatter compensation using the triple-energy window method for single-and dual-isotope SPECT," Journal of Nuclear Medicine, vol. 34, no. 12, pp. 2216-2221, 1993.

[7] A. P. Robinson, J. Tipping, D. M. Cullen, and D. Hamilton, "The influence of triple energy window scatter correction on activity quantification for 177Lu molecular radiotherapy," Physics in Medicine \& Biology, vol. 61, no. 14, p. 5107, 2016.

[8] M. J. Ehrhardt, P. Markiewicz, M. Liljeroth, A. Barnes, V. Kolehmainen, J. S. Duncan, L. Pizarro, D. Atkinson, B. F. Hutton, S. Ourselin, K. Thielemans, and S. R. Arridge, "PET reconstruction with an anatomical MRI prior using parallel level sets," IEEE Transactions on Medical Imaging, vol. 35, no. 9, pp. 2189-2199, 2016.

[9] W. Hutchcroft, G. Wang, K. T. Chen, C. Catana, and J. Qi, "Anatomically-aided PET reconstruction using the kernel method," Physics in Medicine and Biology, vol. 61, no. 18, p. 6668, 2016.

[10] D. Deidda, N. A. Karakatsanis, P. M. Robson, Y. Tsai, N. Efthimiou, K. Thielemans, Z. A. Fayad, R. G. Aykroyd, and C. Tsoumpas, "Hybrid PET-MR list-mode kernelized expectation maximization reconstruction," Inverse Problems, vol. 35, no. 4, pp. 1-24, 2019.

[11] D. Deidda, N. Karakatsanis, C. Calcagno, P. M. Robson, M. Senders, W. J. M. Mulder, Z. A. Fayad, R. G. Aykroyd, and C. Tsoumpas, "Hybrid PET-MR kernelised expectation maximisation reconstruction for improved image- derived estimation of the input function from the aorta of rabbits," Contrast media \& molecular imaging, 2019.

[12] D. Deidda, N. Karakatsanis, P. M. Robson, N. Efthimiou, Z. A. Fayad, R. G. Aykroyd, and C. Tsoumpas, "Effect of PET-MR inconsistency in the kernel image reconstruction method," IEEE Transactions on Radiation and Plasma Medical Sciences, 2019. 